\title{
Population Subdivision and the Frequency of Aflatoxigenic Isolates in Aspergillus flavus in the United States
}

\author{
Milton T. Drott, Lauren M. Fessler, and Michael G. Milgroom ${ }^{\dagger}$
}

Plant Pathology and Plant-Microbe Biology Section, School of Integrative Plant Science, Cornell University, Ithaca, NY 14853 Accepted for publication 23 November 2018.

\begin{abstract}
Consumption of food contaminated with aflatoxin, from crops infected by Aspergillus flavus, is associated with acute toxicosis, cancer, and stunted growth. Although such contamination is more common in the lower latitudes of the United States, it is unclear whether this pattern is associated with differences in the relative frequencies of aflatoxigenic individuals of A. flavus. To determine whether the frequency of the aflatoxin-producing ability of $A$. flavus increases as latitude decreases, we sampled 281 isolates from field soils in two north-south transects in the United States and tested them for aflatoxin production. We also genotyped 161 isolates using 10 microsatellite markers to assess population structure. Although the population density of A. flavus was

highest at lower latitudes, there was no difference in the frequency of aflatoxigenic A. flavus isolates in relation to latitude. We found that the U.S. population of A. flavus is subdivided into two genetically differentiated subpopulations that are not associated with the chemotype or geographic origin of the isolates. The two populations differ markedly in allelic and genotypic diversity. The less diverse population is more abundant and may represent a clonal lineage derived from the more diverse population. Overall, increased aflatoxin contamination in lower latitudes may be explained partially by differences in the population density of A. flavus, not genetic population structure.
\end{abstract}

Mycotoxins contaminate $\sim 25 \%$ of food supplies worldwide (Council for Agricultural Science and Technology 2003) and are associated with human death and disease from acute toxicosis, cancer, immune suppression, and stunted growth in children (Liu and $\mathrm{Wu}$ 2010; Wild 2007; Wild and Gong 2010). An estimated 4.5 billion people are chronically exposed to aflatoxin through contaminated food (Centers for Disease Control and Prevention 2016). Aflatoxin is produced by several species in the genus Aspergillus (section Flavi), with A. flavus being the main producer (Klich 2007). Aflatoxin often contaminates maize, peanuts, cottonseed, tree nuts, and other seed crops. Within A. flavus, there are two morphologically and genetically differentiated phenotypes. These phenotypes were named $\mathrm{S}$ and $\mathrm{L}$ for small and large sclerotia, respectively (Cotty 1989). All S-strain isolates produce aflatoxin, typically in greater quantities than L strain. Populations of $A$. flavus are highly diverse (Bayman and Cotty 1991; Horn and Greene 1995) and, importantly, not all L-strain isolates produce aflatoxin. Extensive field sampling of A. flavus in the United States found that only $71 \%$ of L-strain isolates are aflatoxigenic (Horn and Dorner 1999). Worldwide, both chemotypes - those that produce aflatoxin and those that do not (aflatoxigenic and nonaflatoxigenic, respectively) - are often found in soil in the same field (Horn 2003). There is evidence that competition with insects (Drott et al. 2017) and soil microbes (Drott 2019) may maintain both aflatoxigenic and nonaflatoxigenic individuals by balancing selection. Although aflatoxigenic isolates have been extensively studied because of the aflatoxin contamination they cause,

†Corresponding author: M. G. Milgroom; mgm5@cornell.edu

Funding: This work was supported by the National Institute of Food and Agriculture, U.S. Department of Agriculture award number 2016-67013-24807.

*The $\boldsymbol{e}$-Xtra logo stands for "electronic extra" and indicates that one supplementary figure, four supplementary tables, and one supplementary data set are published online.

The author(s) declare no conflict of interest.

(c) 2019 The American Phytopathological Society nonaflatoxigenic isolates have also received attention for their potential as biocontrol agents. When applied to agricultural fields, nonaflatoxigenic isolates compete with aflatoxigenic individuals, resulting in a reduction of aflatoxin contamination (Ehrlich 2014). Despite the importance of both aflatoxigenic and nonaflatoxigenic isolates, it remains unclear whether the distribution of A. flavus chemotypes correlates with geographic patterns of aflatoxin contamination of crops.

Aflatoxin contamination of crops in the United States is more common in the South (Horn 2007). Several studies have suggested that at lower latitudes, greater contamination is caused by a higher relative frequency of aflatoxigenic individuals (Cotty 1997; Horn 2007; Manabe and Tsuruta 1978; Tran-Dinh et al. 2009; Wicklow and Cole 1982; Wicklow et al. 1994). Consistent with this suggestion, Manabe and Tsuruta (1978) reported a gradient in frequencies with latitude in Japan and Southeast Asia. However, further evidence for this pattern is less clear. Wicklow and Cole (1982) reported a trend toward increased frequency of aflatoxigenic isolates at lower latitudes despite finding no significant difference. Cotty (1997) found a significant negative correlation in the southern United States between latitude and the frequency of aflatoxigenic isolates of the A. flavus L strain. Although their sampling sites (located in Arizona, Arkansas, Alabama, Louisiana, and Mississippi) may have differed somewhat in climate, they differed only by $\sim 3^{\circ}$ of latitude $\left(\sim 34^{\circ}\right.$ to $\left.31^{\circ}\right)$. In Vietnam, Tran-Dinh et al. (2009) also found a significantly higher frequency of aflatoxigenic isolates from peanuts at lower latitudes in Vietnam, but not from corn and soil or when isolates from all substrates were pooled. Furthermore, not all of the evidence supports the prediction of a negative correlation between the frequency of aflatoxin production and latitude. Horn and Dorner (1999) found that the frequency of isolates grouped by amount of aflatoxin produced does not appear to be different in the southern and more northern parts of a transect across the United States. Despite the dearth of evidence that the frequency of aflatoxigenic isolates increases with decreasing latitude, hypotheses have emerged that greater competition with insects and climatic conditions associated with lower latitudes select for aflatoxigenic individuals (Horn 2007; Wicklow et al. 1994). 
Regional differences in the frequency of aflatoxigenic isolates could be maintained by selection, restricted migration, or both. If migration rates were high relative to differences in selective pressures between regions, frequencies of aflatoxigenic individuals may not differ. Previous studies of the population genetics of A. flavus only partially addressed this question. There is some evidence of long-distance migration of A. flavus clones, based on finding isolates of the same vegetative compatibility groups (VCGs) over widespread geographic locations, such as different provinces of Kenya (Probst et al. 2011) or different states in the United States (Ehrlich et al. 2007; Grubisha and Cotty 2010; Horn and Dorner 1999). However, these examples are not definitive, as they either focus on a small subset of VCGs or do not report chemotypes. It is thus not possible to determine whether previous observations of the distribution of VCGs represent infrequent or episodic migration or if migration occurs on a more widespread basis. Making this distinction is necessary for testing the adaptive hypothesis, namely that latitudinal differences in the frequency of aflatoxigenic isolates of $A$. flavus may be maintained by selection.

Comprehensive assessments of migration and population structure in A. flavus have been limited, in part, by the use of VCGs, which are thought to represent clones or clonal lineages in A. flavus populations (Bayman and Cotty 1993; Ehrlich et al. 2007; McAlpin et al. 2002). The usefulness of VCGs as genetic markers, however, is mitigated by several factors, the most important of which are that methods for assaying them are laborious, they exclude large numbers of isolates that are not compatible with known tester isolates, and they do not provide information on genetic relatedness between VCGs. For example, in the most extensive sampling of A. flavus in the United States U.S. to date, Horn and Dorner (1998) initially deemed identification of VCGs too labor intensive, and they later identified only VCGs of nonaflatoxigenic isolates (Horn and Dorner 1999). In addition to being biased only to nonaflatoxigenic isolates, their sample was also limited to more common VCGs, as they were only able to match 73 of 126 isolates to tester isolates. In contrast, studies that have used molecular markers have not been designed to estimate migration of A. flavus. Instead, isolates from culture collections were used in these studies that were not randomly sampled from specific populations (Chang et al. 2006; Geiser et al. 2000), making inferences about migration difficult. Several of the studies looking for evidence of migration focused on small geographic areas (McAlpin et al. 2002; Wicklow et al. 1998). Others did not report whether they found the same genotype in different locations (Barros et al. 2007) or how frequently they found it (Bayman and Cotty 1993; Yin et al. 2009). Tran-Dinh et al. (2009) showed that four of 48 multilocus genotypes (MLGs) sampled from corn collected from markets across Vietnam were found in multiple provinces. However, they did not report which provinces the same genotype was isolated from and did not indicate if they found the same genotype in any of their isolates from peanuts or soil. Although these studies indicate that migration happens on a relatively large geographic scale, it remains unclear how important these events are to population structure.

Phylogenetic studies using molecular markers on A. flavus isolates found that aflatoxigenic and nonaflatoxigenic isolates are often found in the same clades (Barros et al. 2007; Tran-Dinh et al. 1999, 2009), although not in all cases (Baird et al. 2006; Bayman and Cotty 1993). Tran-Dinh et al. (1999) suggest that findings like these indicate that aflatoxin production was lost from aflatoxigenic clonal lineages multiple times, or that recombination has occurred between aflatoxigenic and nonaflatoxigenic lineages. Several studies have found evidence of sexual reproduction in the A. flavus genome, even between aflatoxigenic and nonaflatoxigenic lineages. Such recombination is generally thought to be ancient (Moore et al. 2009, 2013; Olarte et al. 2012). However, some aspects of modern population structure are consistent with recent recombination. Repeated sampling in the same locations or regions has uncovered novel VCGs each time (Bayman and Cotty 1991;
Horn and Greene 1995); this is consistent with recombination between VCGs. There is also evidence that $A$. flavus can reproduce sexually in the field, as a survey of sclerotia produced on inoculated maize ears showed that fertile sexual structures can be found, albeit at a low frequency (Horn et al. 2014). One attempt to look for recent recombination focused on genotyping isolates in a small number of VCGs using microsatellite markers (Grubisha and Cotty 2010). However, mating between VCGs would likely result in recombinants in nonparental VCGs, which were not genotyped, whereas mating within VCGs might be difficult, or impossible, to detect because of limited polymorphisms within any given VCG. Thus, studies looking only within VCGs are unlikely to detect recombination, and studies using molecular markers are needed to better understand the relationship between aflatoxigenic and nonaflatoxigenic lineages.

The overall objective of this study was to determine whether higher levels of aflatoxin contamination in the southern United States than in the northern United States could be explained in part by differences in the frequency of aflatoxigenic isolates. To address whether genetic population structure occurs based on latitude, we sampled A. flavus isolates in two north-south transects in the United States, determined their chemotypes, and genotyped them with microsatellite markers. Specifically, we addressed the following questions for A. flavus populations in the United States: (i) Is the frequency of aflatoxigenic isolates negatively correlated with latitude? (ii) Is the U.S. population of A. flavus genetically subdivided by locations or chemotypes? (iii) Is there evidence that both aflatoxigenic and nonaflatoxigenic MLGs migrate across large geographic distances? (iv) Have nonaflatoxigenic isolates arisen multiple times from aflatoxigenic lineages?

\section{MATERIALS AND METHODS}

Isolation and culturing of $\boldsymbol{A}$. flavus. Soil was collected between 2013 and 2017 from maizefields in an eastern United States transect (Pennsylvania, North Carolina, and Florida) and a central United States transect (Indiana, Iowa, Oklahoma, and Texas) (Fig. 1; Supplementary Table S1). Because of the low density of A. flavus and difficulty in obtaining isolates in the north, samples from Iowa and Indiana were pooled for all analyses except for assessments of migration (see below). Three fields were sampled in 2016 or 2017 in all states except for North Carolina, in which two fields were sampled in 2013 and a third was added in 2017. No two fields were within $1 \mathrm{~km}$ of each other. We used a sampling scheme that minimized the recovery of multiple $A$. flavus isolates from the same fungal ramet. In each field, we collected 25 independent soil samples, each at least $10 \mathrm{~m}$ from any other sample. Each sample contained $\sim 50 \mathrm{~g}$ from the top $2 \mathrm{~cm}$ of soil. Samples were sent from their origins via 2-day shipping and were kept at $4^{\circ} \mathrm{C}$ for less than 3 months before processing.

A. flavus was isolated from soil samples on modified dichloranrose bengal medium using recipes and dilution-plating methods similar to Horn and Dorner (1998). Briefly, $2 \mathrm{~g}$ of soil was suspended in $10 \mathrm{ml}$ of $0.2 \%$ water agar and $100 \mu$ l of this suspension, containing $0.016 \mathrm{~g}$ of soil, was used for each dilution plate. Resulting plates were incubated at $30^{\circ} \mathrm{C}$ for 3 days. Up to three A. flavus colonies were isolated from each soil sample. Conidia from A. flavus colonies were streaked onto $25 \%$-strength Czapek agar (CZA; $2 \%$ agar and $12.25 \mathrm{~g}$ of Czapek medium; Difco Laboratories, Detroit, MI) and incubated for $16 \mathrm{~h}$ at $30^{\circ} \mathrm{C}$ in the dark. For each colony, a single hyphal tip was isolated onto $100 \%$ strength CZA to ensure the presence of only one genotype. Isolates were identified as A. flavus based on morphology (Horn and Dorner 1998). Only A. flavus L-strain isolates, as determined morphologically, were used in this study because they are the most common in the United States. Identification was further confirmed for a subset of 90 isolates against closely related species with similar morphology (e.g., A. nomius) by BLASTn (National Center for 
Biotechnology Information) analysis of $\beta$-tubulin sequences using polymerase chain reaction (PCR) primers designed previously (Tam et al. 2014).

Isolates on CZA plates were inoculated into $20 \mathrm{ml}$ of potato dextrose broth (BD Difco, Franklin Lakes, NJ) in 50-ml tubes and grown for $4 \mathrm{~d}$ at $30^{\circ} \mathrm{C}$. Resulting hyphal mats were harvested by filtration using cheesecloth, rinsed with sterile deionized $\mathrm{H}_{2} \mathrm{O}$, frozen, lyophilized, and stored at $-80^{\circ} \mathrm{C}$ until DNA was extracted (see below).

Colony-forming units of A. flavus were counted for four randomly selected soil samples from every field to estimate population density. Differences in population density between geographic regions were assessed using analysis of variance and a linear contrast to test for latitudinal differences.

Determination of aflatoxin production. All isolates were cultured in yeast extract sucrose medium (Horn and Dorner 1999) and on Drosophila culture medium (DCM) to determine aflatoxin chemotype by high-performance liquid chromatography, as described previously (Drott et al. 2017). Cultures on DCM were mechanically damaged with a sterile toothpick to stimulate greater production of aflatoxin (Drott et al. 2017). Both assays were replicated twice for all isolates. Although A. flavus has the potential to produce a variety of mycotoxins (e.g., cyclopiazonic acid, whose biosynthetic cluster is adjacent to the aflatoxin cluster; Horn et al. 2009), in this article we refer to isolates and their chemotype as aflatoxigenic and nonaflatoxigenic based solely on their ability to produce aflatoxin $\mathrm{B}_{1}$.

The relative frequency of aflatoxigenic isolates in each field was determined from a sample of isolates, all of which originated from independent soil samples. We looked for latitudinal patterns in the frequency of aflatoxigenic isolates using a $\chi^{2}$ test for trends.

DNA extraction and microsatellite genotyping. DNA was extracted from 4-mm-diameter balls of lyophilized mycelium. Tissue was homogenized in a microcentrifuge tube containing 0.5 and $2-\mathrm{mm}$ zirconia-silica beads and $1 \mathrm{ml}$ of extraction buffer (similar to the methods of Kepler et al., 2014) for $6 \mathrm{~s}$ on the 4.5 setting of a Thermo Savant Bio101 Fast Prep 120 homogenizer (Qbiogene, Carlsbad, CA). Extracts were incubated in a boiling water bath for $10 \mathrm{~min}$ and centrifuged for $10 \mathrm{~min}$ at $10,000 \times g$. The supernatant $(100 \mu \mathrm{l})$ was removed and diluted 10 -fold in UltraPure

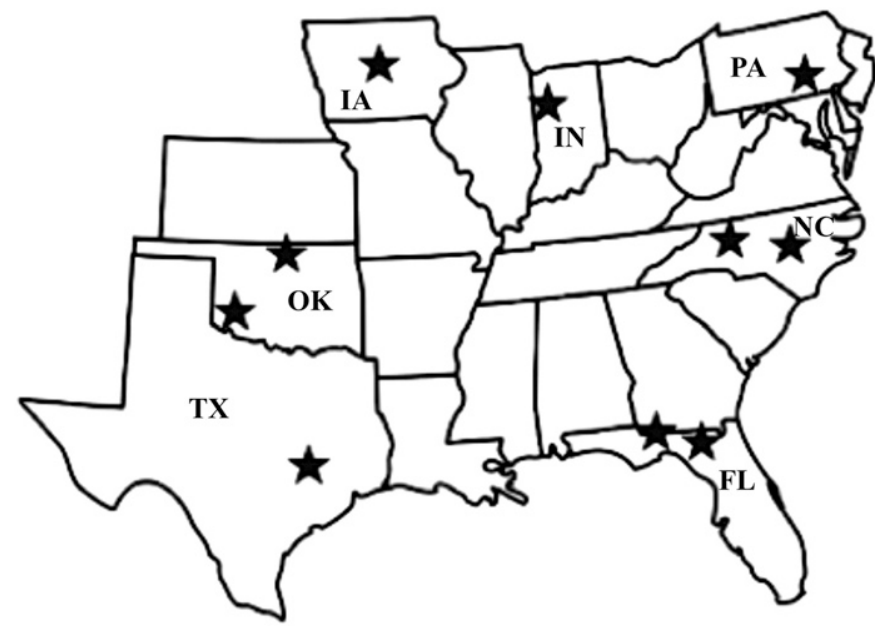

Fig. 1. Sampling locations of two north-south transects in the United States. The eastern transect fields were in Pennsylvania (PA), North Carolina (NC), and Florida (FL). The central transect fields were in Iowa (IA), Indiana (IN), Oklahoma (OK), and Texas (TX). Three fields were sampled in each state; when only one or two stars are present in a state on the map, then more than one field fell within the area covered by the star. No two fields were within $1 \mathrm{~km}$ of each other. The map is modified from a public domain image (https:// commons.wikimedia.org/wiki/File:Blank_US_map_borders.svg). water for use as template DNA for PCR (Thermo Fisher, Waltham, MA).

We selected 10 microsatellite markers described by Grubisha and Cotty (2009) that discriminated among VCGs but had relatively few alleles within VCGs to avoid hypervariable loci. In addition, to maximize the independence of markers, all but four of the markers we selected were located on different genomic scaffolds of the A. flavus genome (Supplementary Table S2). Forward PCR primers were modified by adding a 21-bp M13 tail, allowing for the attachment of fluorophores using methods previously described (Schuelke 2000). Fragments were amplified in $20-\mu \mathrm{l}$ reactions using methods similar to those of Rafiei et al. (2018). Reactions contained $1 \mathrm{U}$ of Native Taq (Thermo Fisher), $400 \mathrm{nM}$ of dNTP mix, $2.5 \mathrm{mM}$ of $\mathrm{MgCl}_{2}, 10 \mu \mathrm{l}$ of $2 \times$ reaction buffer, $1 \mu$ of template DNA (see above), $250 \mathrm{nM}$ of reverse primer, $50 \mathrm{nM}$ of forward primer with the M13 tail, and $200 \mathrm{nM}$ of M13 primer labeled with a fluorophore (either NED, VIC, or FAM). Amplification conditions were as follows: $95^{\circ} \mathrm{C}$ for $2 \mathrm{~min}$; 35 cycles of $95^{\circ} \mathrm{C}$ for $10 \mathrm{~s}, 58^{\circ} \mathrm{C}$ for $20 \mathrm{~s}$, and $72^{\circ} \mathrm{C}$ for $40 \mathrm{~s}$; and a final incubation at $72^{\circ} \mathrm{C}$ for $5 \mathrm{~min}$. Up to three PCR products labeled with different fluorophores originating from the same isolate were pooled by combining $3 \mu \mathrm{l}$ of each. Pooled samples were mixed with $0.2 \mu \mathrm{l}$ of LIZ500 size standard (Applied Biosystems, Foster City, CA) and $10 \mu \mathrm{l}$ of highly deionized formamide (Hi-Di formamide; Applied Biosystems). This mixture was denatured at $95^{\circ} \mathrm{C}$ for $5 \mathrm{~min}$. Fragment sizes were measured using capillary electrophoresis on an ABI 3730xl DNA Analyzer (Applied Biosystems) at Cornell University Life Sciences Core Laboratories. Resulting fragment sizes were determined using Peak Scanner software (Applied Biosystems).

To increase sample sizes of northern sampling locations where we obtained fewer isolates, multiple isolates originating from the same soil sample were genotyped. In cases in which two isolates yielded an identical MLG, only one of them was retained to avoid sampling the same ramet twice. At all other sampling locations only a single isolate was retained from each independent soil sample.

Analysis of population structure. Allelic diversity was calculated as $1-\sum p_{i}^{2}$, where $p_{i}$ is the frequency of the $i$ th allele at each microsatellite locus and averaged across 10 loci (Nei 1987). Genotypic diversity, based on frequencies of MLGs, was estimated as the Simpson index using poppr version 2.5.1 (Kamvar et al. 2014) in $\mathrm{R}$ statistics 3.4.0 ( $\mathrm{R}$ Core Team 2017). The genetic relatedness of MLGs was determined by Bruvo's distance and visualized in minimum-spanning networks (MSNs) using poppr. As an alternative to MSNs, we also show genetic relationships among MLGs in a neighbor-network created using SplitsTree version 4.14.6 (Huson and Bryant 2005). Data were grouped into a priori populations based on state or chemotype and tested for population subdivision using analysis of molecular variance (AMOVA) executed in GENALEX version 6.5 (Peakall and Smouse 2006). Population structure was also analyzed a posteriori using the nonmodel-based multivariate analysis discriminant analysis of principal components (DAPC) (Jombart 2008) according to procedures outlined in the adegenet $\mathrm{R}$ package tutorial (Jombart 2015). Genetic isolation by distance between states was explored using a Mantel test with 1,000 permutations in the ade4 R package (Dray and Dufour 2007).

Analysis of recombination. To look for evidence of recombination in A. flavus populations, we estimated linkage disequilibrium (LD). We calculated the index of association $\left(I_{\mathrm{A}}\right)$ and the standardized index of association $\left(\bar{r}_{\mathrm{d}}\right)$ in poppr on clonecorrected data sets to determine whether A. flavus populations significantly deviated from random mating. $I_{\mathrm{A}}$ and $\bar{r}_{\mathrm{d}}$ are used as measures of multilocus LD among all loci simultaneously, avoiding issues of multiple comparisons often associated with estimates of LD between pairs of loci. $\bar{r}_{\mathrm{d}}$ can be interpreted as a correlation coefficient, scaled between 0 and 1 . Significance for $I_{\mathrm{A}}$ (and $\bar{r}_{\mathrm{d}}$ ) was determined by comparison with estimates of $I_{\mathrm{A}}$ from 1,000 random permutations that simulated random mating.

We calculated the probability that isolates with the same MLG arose independently by recombination $\left(P_{\mathrm{sex}}\right)$ with poppr using the 
multiple comparison method. This method utilizes binomial equations from Arnaud-Haond et al. (2007) to calculate the probability of finding one more individual with the same MLG as an MLG already in the sample.

We also looked for evidence of recombination using the fourgamete test (Hudson and Kaplan 1985). The presence of all four two-locus genotypes (each locus with two alleles) is known as phylogenetic incompatibility in clonal populations (Anderson and Kohn 1998) and can arise either by recombination or by parallel or reverse mutations. Pairwise comparisons of all 10 loci used to determine MLGs were assessed for phylogenetic incompatibility using the R package FourgameteP developed in this study (Drott 2018).

General statistical methods. Results were analyzed using R statistics 3.4.0 (R Core Team 2017) and packages poppr version 2.5.1 (Kamvar et al. 2014), lme4 (Bates et al. 2015), car (Fox and Weisberg 2011), lmerTest (Kuznetsova et al. 2016), and lsmeans (Lenth 2016) installed on 21 April 2017. Graphing of results was also completed using $\mathrm{R}$ statistics with the packages tidyverse (Wickham 2017) and Rmisc (Hope 2013) installed on the same day.

\section{RESULTS}

Population density and frequency of aflatoxigenic isolates in relation to latitude. The population density of A. flavus in soil (expressed as colony-forming unit counts) increased significantly from north to south $(P<0.001)$. In both the eastern and central transects, the highest densities were in the most southerly populations (Florida and Texas), whereas densities were very low in the most northerly populations (Pennsylvania, Indiana, and Iowa) and intermediate in the middle (North Carolina and Oklahoma) (Fig. 2). Population density increased 100-fold on average from north to south. There was, however, large variation in density even within states, as can be seen by the relatively large standard errors in Figure 2. In Florida, for example, fields had averages of 5,000, 3,031 , and $78 \mathrm{CFU} / \mathrm{g}$ of soil. Similar variation was also seen in Texas and North Carolina.

We obtained 281 isolates of A. flavus, each from an independent soil sample, across both transects (Fig. 1). Sequences of $\beta$-tubulin from 90 isolates identified morphologically as A. flavus L strain confirmed their identities. The frequency of aflatoxigenic isolates

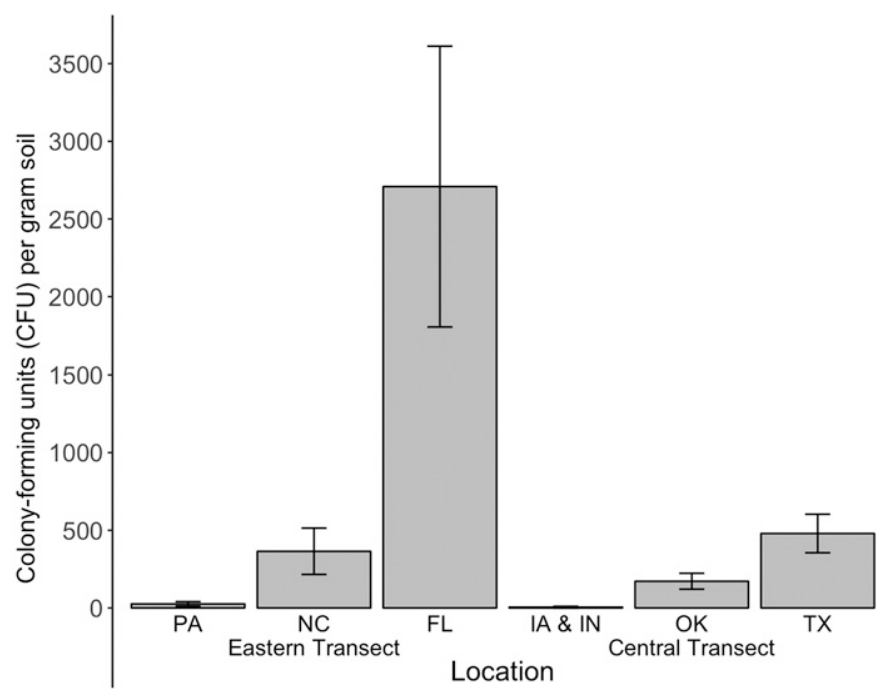

Fig. 2. Average population density of Aspergillus flavus, expressed as colonyforming units per gram of soil, in two north-south transects in the United States. Colony-forming units were averaged across four soil samples for each of three fields per state. Error bars are $\pm 1 \mathrm{SE}$ for variation among fields within states. $\mathrm{PA}=$ Pennsylvania, $\mathrm{NC}=$ North Carolina, $\mathrm{FL}=$ Florida, $\mathrm{IA}=$ Iowa, $\mathrm{IN}=$ Indiana, $\mathrm{OK}=$ Oklahoma, and $\mathrm{TX}=$ Texas did not increase significantly with decreasing latitude in either transect $(P>0.61)$. Indeed, contrary to predictions, the frequency of aflatoxigenic isolates increased with latitude $(P=0.023)$ in the central transect (Fig. 3). Although variation in the frequency of aflatoxigenic isolates was high at the state level, the eastern and central transects were similar when states were pooled within transects, with frequencies of 0.34 and 0.39 aflatoxigenic isolates, respectively (Fig. 3).

Population subdivision and migration. We obtained genotypic data on 168 isolates, seven of which were discarded because they originated from a soil sample where an identical MLG had already been sampled (Supplementary Data Set S1). The remaining 161 independent isolates represented 102 unique MLGs, eight of which originated from the same soil sample as another isolate with a different MLG. Closely related isolates were often from different states and/or different chemotypes (Fig. 4).

The U.S. population of A. flavus was subdivided into two subpopulations. Analysis of underlying population structure, without a priori assumptions, from DAPC inferred two populations $(k=2)$. AMOVA of the two populations identified by DAPC also showed significant differentiation $\left(\Phi_{\mathrm{PT}}=0.244, P=0.001\right)$. One population was characterized by distantly related MLGs (population A), whereas the other comprised more closely related MLGs (population B); this difference is evident from the thicker lines separating MLGs in population B than in population A in the MSN (Fig. 4), indicating smaller genetic distances in population B. Moreover, allelic and genotypic diversities were higher in population $\mathrm{A}$ than in population $\mathrm{B}$ and the clonal fraction of population A (0.041) was markedly lower than that of population $B$ (0.509) (Table 1). The frequency of aflatoxigenic isolates was 0.57 in population $\mathrm{A}$, whereas this same frequency was only 0.30 in population B; with clone correction, these frequencies were 0.57 and 0.35 , respectively (Table 1 ).

Because of significant differentiation between populations A and $\mathrm{B}$, their population structures were analyzed separately. We found no evidence in AMOVA for genetic differentiation among states for either population $\mathrm{A}\left(\Phi_{\mathrm{PT}}=0, P=0.361\right)$ or population $\mathrm{B}\left(\Phi_{\mathrm{PT}}=\right.$ $0.005, P=0.347$ ) (Table 1). Consistent with the lack of genetic differentiation among states, we found nine MLGs of population B in two or more states. In population A, we only found isolates that shared an MLG twice and both were from the same state (Fig. 4A).

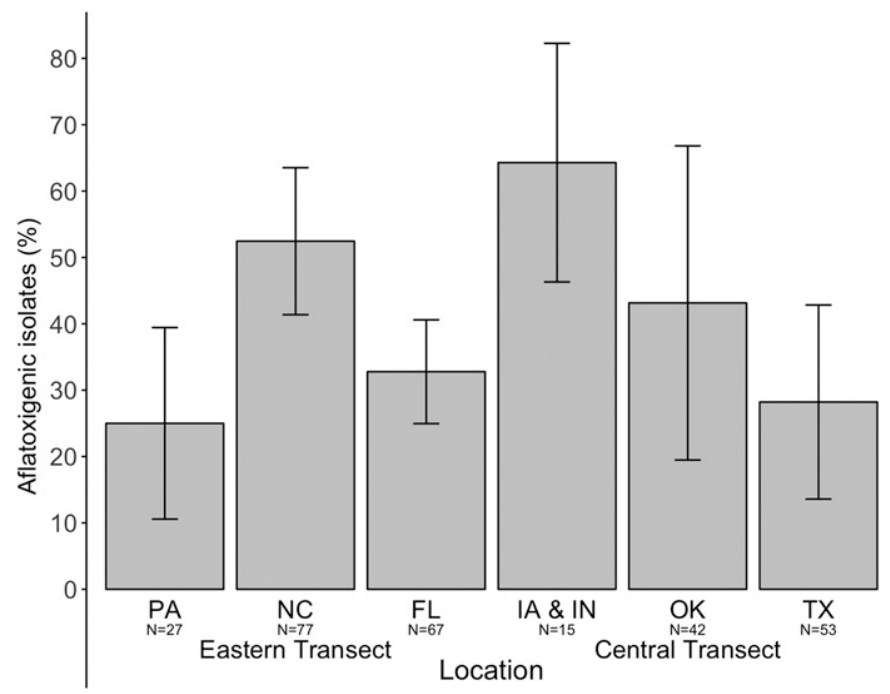

Fig. 3. The relative frequencies of aflatoxigenic isolates of Aspergillus flavus in two north-south transects in the United States. The frequency of aflatoxigenic isolates was determined from samples of isolates from independent soil samples within each state, with Iowa and Indiana pooled. Error bars are $\pm 1 \mathrm{SE}$ for variation among fields within states. PA = Pennsylvania, $\mathrm{NC}=$ North Carolina, $\mathrm{FL}=$ Florida, $\mathrm{IA}=$ Iowa, $\mathrm{IN}=$ Indiana, $\mathrm{OK}=$ Oklahoma, and $\mathrm{TX}=$ Texas. 
In population B, seven MLGs were found in two states (six in North Carolina and Florida, and one in Florida and Texas); one MLG was found in three states (Florida, Oklahoma, and Texas) and another MLG was found in four states (Indiana, Iowa, Texas, and Oklahoma) (Fig. 4A). These results indicate that clonal migration has occurred between states in population B, but we found no evidence of this in population A. In contrast, the Mantel test indicated significant isolation by distance between states for population A $(r=0.186, P<0.001)$ and population B $(r=0.094$, $P<0.001$ ) (Table 1). Altogether, these results suggest some degree

A

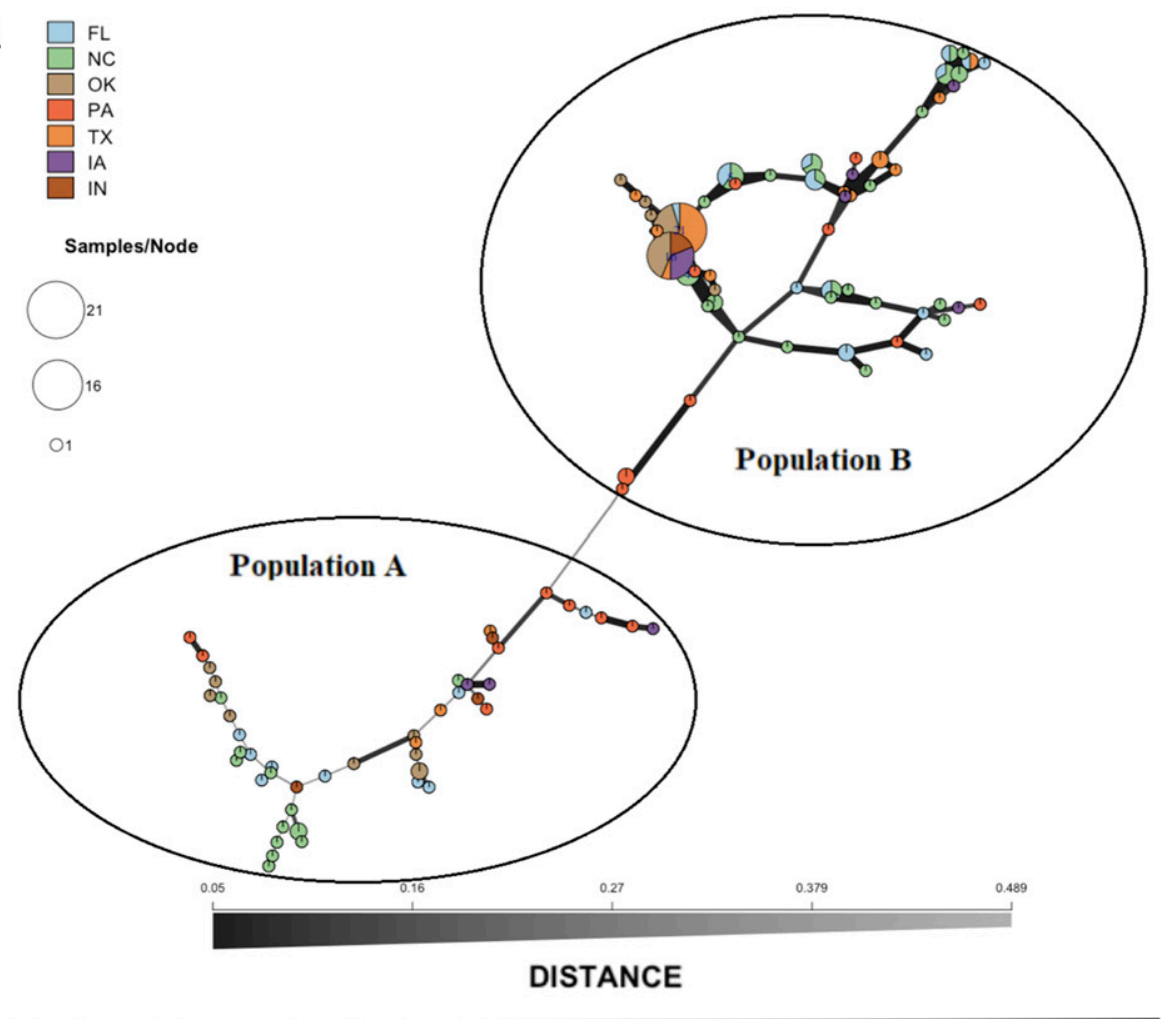

B

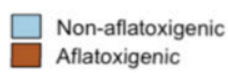

Samples/Node

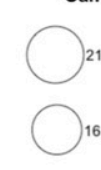

O1

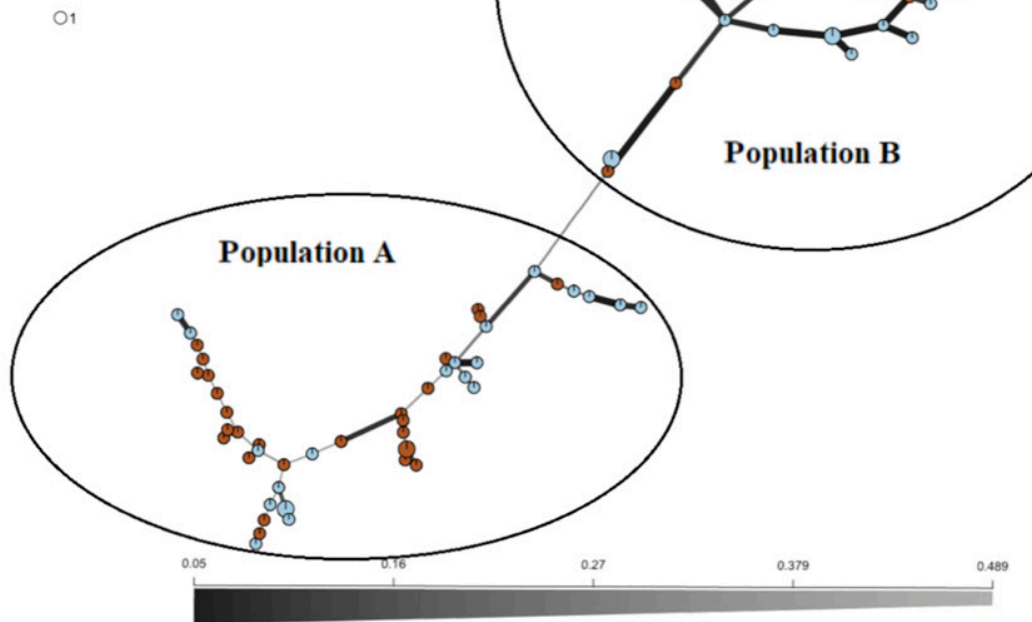

DISTANCE

Fig. 4. Minimum-spanning network representing the genetic relatedness of multilocus genotypes of 161 Aspergillus flavus isolates sampled from two north-south transects in the United States. Isolates were categorized by A, state of origin and $\mathbf{B}$, chemotype. Assignment of isolates to populations A and B was made by discriminant analysis of principal components. Note the thin line separating populations A and B, indicating a large genetic distance. Genetic distances were estimated from 10 microsatellite markers using Bruvo's distance. $\mathrm{FL}=$ Florida, $\mathrm{NC}=$ North Carolina, $\mathrm{OK}=\mathrm{Oklahoma}, \mathrm{PA}=\mathrm{Pennsylvania}$, TX $=$ Texas, IA = Iowa, and IN = Indiana. 
of restricted migration in both populations, but also some evidence of long-distance migration of MLGs between states in population B.

Mantel tests indicated that the average genetic distance within chemotypes was smaller than between chemotypes for population A $(r=0.063, P=0.007)$, but not for population B $(r=0.031, P=$ 0.244) (Table 1). Consistent with this finding, the AMOVA comparing chemotypes indicated a small but significant partitioning of genetic diversity between chemotypes of population $\mathrm{A}\left(\Phi_{\mathrm{PT}}=\right.$ $0.065, P<0.001)$, but not of population B $\left(\Phi_{\mathrm{PT}}=0, P=0.470\right)$ (Table 1). These results indicate that individuals of both chemotypes in population $\mathrm{A}$, but not population $\mathrm{B}$, are genetically more closely related to other individuals in the same chemotype than they are to those in the other chemotype.

Isolates with the same MLG also had the same chemotype, with the exception of two MLGs in population B (Fig. 4B). One of these MLGs (from North Carolina) had a single nonaflatoxigenic isolate and four aflatoxigenic isolates. We also observed an MLG containing eight aflatoxigenic isolates and nine nonaflatoxigenic isolates. In this latter MLG, all toxigenic isolates and a single nonaflatoxigenic isolate were from Iowa or Indiana; the remaining nonaflatoxigenic isolates in this MLG were from Texas or Oklahoma. In population A, the isolates in each of two MLGs with multiple isolates were consistent for chemotype. Finding both chemotypes in the same MLG in population B suggests that nonaflatoxigenic individuals arose by mutation within a predominantly aflatoxigenic lineage.

Recombination. Two MLGs in population A were each represented by two isolates, making up 4\% (clonal fraction 0.04) of the sample, whereas 15 MLGs were represented by two or more isolates in population $\mathrm{B}$, making up $51 \%$ (clonal fraction 0.51 ) of the sample (Fig. 4; Table 1). The probability that we would sample an MLG more than once in isolates that arose independently as a result of random mating and recombination $\left(P_{\text {sex }}\right)$ was $<0.001$ for both clonal MLGs in population A and ranged from $<0.001$ to 0.361 for the 15 clonal MLGs in population B (Table 1). Low estimates of $P_{\text {sex }}$ (e.g., $<0.05$ ) are interpreted as evidence of clonality, whereas higher estimates cannot rule out the possibility that some MLGs could have arisen by recombination. Because estimates of $P_{\text {sex }}$ for five MLGs in population B are less than 0.05, including two of the most common MLGs found in multiple states, we interpret finding the same MLG in different states as indicating evidence of long-distance migration of clones.

Estimates of $I_{\mathrm{A}}$ were significantly different from expectations of random mating for both populations $\mathrm{A}$ and $\mathrm{B}$ although $\bar{r}_{\mathrm{d}}$ was small, indicating weak $\operatorname{LD}\left(\bar{r}_{\mathrm{d}}=0.0526, P<0.001 ; \bar{r}_{\mathrm{d}}=0.0289, P=0.004\right.$, respectively) (Table 1 ). Low levels of LD are consistent with a clonal, or partially clonal, population structure but do not rule out recombination. In this regard, phylogenetic incompatibility (determined by the four-gamete test) was observed at all 45 pairwise comparisons between loci for population A. In population B, however, phylogenetic incompatibility was found at only 22 of the 45 pairwise comparisons (Table 1). These differences in phylogenetic incompatibility between the two populations are also evident in a neighbor-network analysis, in which we observed many more closed loops among MLGs in population A than in population B (Supplementary Fig. S1). In this analysis, unlike MSNs, closed loops are indicative of phylogenetic incompatibility, which can be caused either by recombination or by parallel or reverse mutations. We cannot distinguish between these explanations with our data because we observed a relatively large number of alleles per locus. The mean number of alleles per locus in population A was 9.8 in a sample of 49 (Table 1; Supplementary Table S3), whereas half the number of alleles per locus were found in population $\mathrm{B}$ (mean allelic richness 3.8) in a sample twice the size $(n=112$; Table 1 ; Supplementary Table S4). The high allelic richness in population A raises the possibility that high mutation rates at these loci could result in parallel or reverse mutations. This interpretation is explained in more detail in the next section.

\section{DISCUSSION}

The primary objective of this study was to test the hypothesis that higher levels of aflatoxin contamination in warmer, lower latitudes of the United States (Cotty and Jaime-Garcia 2007) were associated with higher relative frequencies of aflatoxigenic isolates in the A. flavus population. This hypothetical association led to speculations that aflatoxigenic fungi are adapted to warmer climates and increased abundance of insects and that a latitudinal gradient would be maintained by selection (Horn 2007; Wicklow et al. 1994). Although our eastern and central transects spanned $11.5^{\circ}\left(42.1^{\circ}\right.$ to $\left.30.6^{\circ}\right)$ and $9.6^{\circ}\left(40.2^{\circ}\right.$ to $\left.30.6^{\circ}\right)$ of latitude, respectively, we did not

TABLE 1. Comparison of diversity, population structure, and recombination between population A and population B

\begin{tabular}{|c|c|c|}
\hline Statistics & Population A & Population B \\
\hline Sample size, $n$ & 49 & 112 \\
\hline Mean allelic diversity ${ }^{a}$ & 0.780 & 0.296 \\
\hline Total alleles at all loci & 98 & 38 \\
\hline Number of MLGs observed $(g)$ & 47 & 55 \\
\hline Clonal fraction ${ }^{\mathrm{c}}$ & 0.041 & 0.509 \\
\hline Population subdivision $\left(\Phi_{\mathrm{PT}}\right)$ : chemotype ${ }^{\mathrm{d}}$ & $0.059(<0.001)^{\mathrm{e}}$ & $0(0.721)$ \\
\hline Population subdivision $\left(\Phi_{\mathrm{PT}}\right)$ : state ${ }^{\mathrm{d}}$ & $0(0.374)$ & $0.005(0.285)$ \\
\hline Relative frequency of aflatoxigenic isolates & 0.57 & 0.30 \\
\hline Relative frequency of aflatoxigenic MLGs (clone corrected) & 0.57 & $0.35^{\mathrm{g}}$ \\
\hline Linkage disequilibrium $\left(I_{\mathrm{A}} \mathrm{h}^{\mathrm{h}}\right.$ & $0.454(<0.001)$ & $0.235(0.004)$ \\
\hline Linkage disequilibrium $\left(\bar{r}_{\mathrm{d}}\right)^{\mathrm{h}}$ & $0.0526(<0.001)$ & $0.0289(0.004)$ \\
\hline Phylogenetic incompatibility (four-gamete test) ${ }^{\mathrm{i}}$ & 45 & 22 \\
\hline
\end{tabular}

a Allelic diversity was estimated for each locus as $1-\sum p_{1}^{2}$, where $p_{\mathrm{i}}$ is the relative frequency of the $i$ th allele within a population, and averaged across 10 loci.

${ }^{\mathrm{b}}$ Genotypic diversity was estimated as $1-\Sigma p_{1}^{2}$, where $p_{\mathrm{i}}$ is the relative frequency of the $i$ th multilocus genotype (MLG).

c Clonal fraction was estimated as $1-g / n$, where $g$ is the number of MLGs observed and $n$ is the sample size.

d Population subdivision was estimated using analysis of molecular variance (see the text).

e $P$ values are shown in parentheses.

${ }^{\mathrm{f}}$ Isolation by distance was estimated using the Mantel test (see the text).

$\mathrm{g}$ This frequency was based on finding aflatoxigenic isolates in 20 MLGs and nonaflatoxigenic isolates in 27 MLGs. Both chemotypes were found in two MLGs; these two MLGs were therefore counted twice for calculating relative frequency: $0.35=20 / 57$.

${ }^{\mathrm{h}}$ Linkage disequilibrium was estimated by calculating the index of association $\left(I_{\mathrm{A}}\right)$ and the standardized index of association $\left(\bar{r}_{\mathrm{d}}\right)$ (see the text).

i Number of locus pairs showing phylogenetic incompatibility across all 45 pairs of polymorphic loci (see the text). 
observe any evidence of a correlation between frequency of aflatoxigenic A. flavus and latitude (Fig. 3). Therefore, our results do not support the hypothesis that selection maintains a geographic pattern (Cotty 1997; Horn 2007; Wicklow and Cole 1982; Wicklow et al. 1994). Rather, our findings are consistent with those of Horn and Dorner (1999), who found no clear latitude-related pattern in the frequency of isolates producing different amounts of aflatoxin. Their transect, however, was characterized by sampling mostly east to west ( $\sim 28^{\circ}$ longitude) in the southern United States and captured less variation in latitude $\left(\sim 6.5^{\circ}\right.$, from $\sim 31.5$ to $\left.\sim 38\right)$ than our study. In contrast, our study examined two replicated north-south transects including a range of conditions, from those reported to have relatively little aflatoxin contamination to those with high levels of contamination. Our study is a relatively comprehensive assessment across latitudes, and yet we found no differences in the frequency of aflatoxigenic isolates.

In contrast to the frequency of aflatoxigenic isolates, the overall density of A. flavus in soil (in colony-forming units per gram) was very low in higher latitudes relative to lower latitudes (Fig. 2). Despite repeated attempts, we were unable to isolate $A$. flavus from the soil of several fields in Ithaca and Aurora, New York (both at $\sim 42.5^{\circ}$ latitude) (data not shown). Indeed, our results suggest a northern limit to the range of A. flavus in the United States, although there are some reports of the fungus at higher latitudes than those sampled here (Klich 2002). The geographic patterns we found for population density are consistent with a suppressive soil effect observed by Drott (2019) under laboratory conditions: namely, soils incubated at cooler temperatures are more inhibitory to the growth of A. flavus than those incubated at warmer temperatures. At lower latitudes, stress on crops associated with both drought (Jones et al. 1981) or high temperature (Abbas et al. 2002) results in greater aflatoxin contamination. Consistent with these observations, Cotty and Jaime-Garcia (2007) suggested that crops grown in warmer climates are more likely to be infected by A. flavus and have greater aflatoxin contamination. We speculate that greater aflatoxin contamination at lower latitudes (Horn 2007) is likely caused by an interaction between the higher population density of $A$. flavus and the effect of climate in warmer, lower latitudes on the susceptibility of crops to aflatoxin contamination.

As we did not see any geographic pattern in the frequency of aflatoxigenic isolates (Fig. 3), we also observed no population structure based on microsatellite genotyping that could be explained by geography. This lack of differentiation among states could be explained by migration. We found direct evidence of migration in population B because nine MLGs, accounting for more than half of the isolates, were sampled from more than one state (Fig. 4A). By contrast, in population A we did not observe any MLG in more than one state. Given the higher allelic diversity, lower clonal fraction, and smaller sample size in population A (Table 1), this difference may reflect the low probability of sampling the same MLG twice. Both populations had weak but significant isolation by distance, suggesting some degree of restricted migration. Migration over large geographic areas by aerial dispersal is commonly observed in some plant pathogenic fungi (Brown and Hovmøller 2002). A. flavus has potential for long-distance aerial dispersal, based on the size (Horn et al. 2001) and production of large numbers of conidia that can survive for years under field conditions (Wicklow et al. 1993). Human-mediated migration may also contribute to the patterns of migration we observed, as demonstrated in other plant pathogenic fungi (Linde et al. 2009). Overall, our study suggests that migration of both A. flavus chemotypes is common, despite some restriction evident from significant isolation by distance.

Weak evidence of population structure in population A and the lack of population structure in population B based on chemotype suggests than nonaflatoxigenic genotypes have arisen independently multiple times by mutation or recombination. Previous studies concluded that nonaflatoxigenic isolates descended from aflatoxigenic genotypes in the evolutionary history of $A$. flavus, as indicated by the presence of partial aflatoxin gene clusters (Chang et al. 2005). Examination of the MSN color coded for chemotype (Fig. 4B) shows a mosaic of aflatoxigenic and nonaflatoxigenic isolates throughout the networks in both populations A and B, including aflatoxigenic and nonaflatoxigenic isolates within the same MLG in population B. Other studies also found isolates of different chemotypes in the same phylogenetic clades (Barros et al. 2007; Tran-Dinh et al. 1999, 2009). These findings serve to emphasize that aflatoxigenic and nonaflatoxigenic isolates may be very closely related and suggest that nonaflatoxigenic isolates may arise relatively frequently in extant populations.

The role of recombination in the origin of nonaflatoxigenic genotypes remains unclear. Although some aspects of A. flavus population structure are consistent with clonality, other evidence is consistent with recombination. In this study, we found phylogenetic incompatibility (using the four-gamete test) in all 45 pairwise comparisons of 10 loci for population A, but only in 25 of 45 comparisons for population B. The four-gamete test is used for detecting recombination but depends on the crucial assumption that each allele is unique and has arisen by mutation only once, such that alleles that are identical in state are also identical by descent (Hudson and Kaplan 1985). Even though we chose microsatellite markers with relatively few alleles and minimal polymorphism within VCGs (Grubisha and Cotty 2009), we found an average of 9.8 alleles per locus in a sample of 49 isolates in population A. This level of allelic richness raises the possibility that the assumption of unique mutations has been violated. Such allelic richness is likely to arise by relatively high mutation rates, increasing the probability that some alleles of the same size (alleles are scored by the length of the PCR fragment), although identical in state, arise more than once by parallel or reverse mutation and are therefore not identical by descent (Estoup et al. 2002). This results in the detection of phylogenetic incompatibility in the four-gamete test in the absence of recombination. Thus, although our results raise questions about the role of recombination in the two populations we identified, we cannot confidently determine whether we observed phylogenetic incompatibility because of recombination or parallel or reverse mutations; therefore, we cannot infer whether some nonaflatoxigenic genotypes arose by recombination.

Greater allelic diversity in population A than population B (Table 1) underlies many of the differences we observed between these two populations, affecting genotypic diversity, clonal fraction, and phylogenetic incompatibility. We speculate that population B is a clonal lineage derived from population A (Fig. 4), with high fitness and therefore commonly sampled from soil in agricultural fields. In contrast, population A represents much of the diversity within the population of A. flavus L strains we sampled in the United States. In this regard, A. flavus population structure is consistent with an epidemic population structure (Smith et al. 1993), in which one or a few highly fit genotypes become the dominant clones in an otherwise diverse population. Additional sampling and/or genotyping with markers such as single nucleotide polymorphisms with lower mutation rates than microsatellites will be needed to confirm this population structure.

Our results help to explain patterns of aflatoxin contamination in the United States while also providing information on the population structure of $A$. flavus that informs efforts to mitigate contamination. Our interpretation is that greater aflatoxin contamination in the southern United States (Horn 2007) is more likely related to higher population densities of A. flavus than to higher frequencies of aflatoxigenic isolates. This trend in population density is consistent with increased suppression of $A$. flavus growth in soils incubated at cooler temperatures (Drott 2019). The population structuring we observed could not readily be explained by differences in geography or chemotype. We further speculate that differences between the two populations may be explained by the divergence of a highly fit clonal lineage, which we called 
population B. Regardless of what may have driven the genetic differentiation of these two populations, it does not appear to be related to chemotype. Both populations contained aflatoxigenic and nonaflatoxigenic isolates with no discernable pattern across geographic locations. Our results suggest that selective pressures acting on aflatoxin production occur in both populations at the field level instead of on a broad geographic scale as previously suggested (Cotty 1997; Horn 2007; Manabe and Tsuruta 1978; Tran-Dinh et al. 2009; Wicklow and Cole 1982; Wicklow et al. 1994). Indeed, competition between A. flavus and insects (Drott et al. 2017) or soil microbes (Drott 2019) may maintain both chemotypes on a local scale. We speculate that even when selective pressures in a local area favor one chemotype, migration may maintain both chemotypes locally. Future work that accounts for the observed population subdivision is needed to better define differences between these two populations, particularly with respect to recombination and migration.

\section{LITERATURE CITED}

Abbas, H. K., Williams, W. P., Windham, G. L., Pringle, H. C., Xie, W., and Shier, W. T. 2002. Aflatoxin and fumonisin contamination of commercial corn (Zea mays) hybrids in Mississippi. J. Agric. Food Chem. 50: 5246-5254.

Anderson, J. B., and Kohn, L. M. 1998. Genotyping, gene genealogies and genomics bring fungal population genetics above ground. Trends Ecol. Evol. 13:444-449.

Arnaud-Haond, S., Duarte, C. M., Alberto, F., and Serrao, E. A. 2007. Standardizing methods to address clonality in population studies. Mol. Ecol. 16: 5115-5139.

Baird, R., Trigiano, R., Windham, G., Williams, P., Kelley, R., Abbas, H., Moulton, J., and Scruggs, M. 2006. Comparison of aflatoxigenic and nonaflatoxigenic isolates of Aspergillus flavus using DNA amplification fingerprinting techniques. Mycopathologia 161:93-99.

Barros, G., Chiotta, M., Reynoso, M., Torres, A., and Chulze, S. 2007. Molecular characterization of Aspergillus section Flavi isolates collected from peanut fields in Argentina using AFLPs. J. Appl. Microbiol. 103:900-909.

Bates, D., Mächler, M., Bolker, B., and Walker, S. 2015. Fitting linear mixedeffects models using lme4. J. Stat. Softw. 67:48.

Bayman, P., and Cotty, P. J. 1991. Vegetative compatibility and genetic diversity in the Aspergillus flavus population of a single field. Can. J. Bot. 69: 1707-1711.

Bayman, P., and Cotty, P. J. 1993. Genetic diversity in Aspergillus flavus association with aflatoxin production and morphology. Can. J. Bot. 71:23-31.

Brown, J. K., and Hovmøller, M. S. 2002. Aerial dispersal of pathogens on the global and continental scales and its impact on plant disease. Science 297: 537-541.

Centers for Disease Control and Prevention. 2016. Health Studies Branch. Understanding chemical exposures: Aflatoxin. https://www.cdc.gov/nceh/ hsb/chemicals/aflatoxin.htm

Chang, P.-K., Ehrlich, K. C., and Hua, S.-S. T. 2006. Cladal relatedness among Aspergillus oryzae isolates and Aspergillus flavus S and L morphotype isolates. Int. J. Food Microbiol. 108:172-177.

Chang, P. K., Horn, B. W., and Dorner, J. W. 2005. Sequence breakpoints in the aflatoxin biosynthesis gene cluster and flanking regions in nonaflatoxigenic Aspergillus flavus isolates. Fungal Genet. Biol. 42:914-923.

Cotty, P. J. 1989. Virulence and cultural characteristics of two Aspergillus flavus strains pathogenic on cotton. Phytopathology 79:808-814.

Cotty, P. J. 1997. Aflatoxin-producing potential of communities of Aspergillus section Flavi from cotton producing areas in the United States. Mycol. Res. 101:698-704.

Cotty, P. J., and Jaime-Garcia, R. 2007. Influences of climate on aflatoxin producing fungi and aflatoxin contamination. Int. J. Food Microbiol. 119: $109-115$.

Council for Agricultural Science and Technology. 2003. Mycotoxins: Risk in Plant, Animal, and Human Systems. Council for Agricultural Science and Technology, Ames, IA.

Dray, S., and Dufour, A.-B. 2007. The ade4 package: Implementing the duality diagram for ecologists. J. Stat. Softw. 22:1-20.

Drott, M. T., Debenport, T., Higgins, S. A., Buckley, D. H., and Milgroom, M. G. 2019. Fitness cost of aflatoxin production in Aspergillus flavus when competing with soil microbes could maintain balancing selection. Mbio. 10: e02782-18.

Drott, M. T. 2018. FourGamete test. R package version 0.1.0.

Drott, M. T., Lazzaro, B. P., Brown, D. L., Carbone, I., and Milgroom, M. G. 2017. Balancing selection for aflatoxin in Aspergillus flavus is maintained through interference competition with, and fungivory by insects. Proc. Biol. Sci. 284:20172408.

Ehrlich, K. C. 2014. Non-aflatoxigenic Aspergillus flavus to prevent aflatoxin contamination in crops: Advantages and limitations. Front. Microbiol. 5: $1-9$.

Ehrlich, K. C., Montalbano, B. G., and Cotty, P. J. 2007. Analysis of single nucleotide polymorphisms in three genes shows evidence for genetic isolation of certain Aspergillus flavus vegetative compatibility groups. FEMS Microbiol. Lett. 268:231-236.

Estoup, A., Jarne, P., and Cornuet, J. M. 2002. Homoplasy and mutation model at microsatellite loci and their consequences for population genetics analysis. Mol. Ecol. 11:1591-1604.

Fox, J., and Weisberg, S. 2011. An R Companion to Applied Regression, 2nd ed. SAGE, Thousand Oaks, CA.

Geiser, D. M., Dorner, J. W., Horn, B. W., and Taylor, J. W. 2000. The phylogenetics of mycotoxin and sclerotium production in Aspergillus flavus and Aspergillus oryzae. Fungal Genet. Biol. 31:169-179.

Grubisha, L., and Cotty, P. 2009. Twenty-four microsatellite markers for the aflatoxin-producing fungus Aspergillus flavus. Mol. Ecol. Resour. 9: 264-267.

Grubisha, L. C., and Cotty, P. J. 2010. Genetic isolation among sympatric vegetative compatibility groups of the aflatoxin-producing fungus Aspergillus flavus. Mol. Ecol. 19:269-280.

Hope, R. M. 2013. Rmisc: Ryan Miscellaneous. R package version 1.5.

Horn, B., and Dorner, J. 1998. Soil populations of Aspergillus species from section Flavi along a transect through peanut-growing regions of the United States. Mycologia 90:767-776.

Horn, B., Greene, R., Sorensen, R., Blankenship, P., and Dorner, J. 2001. Conidial movement of nontoxigenic Aspergillus flavus and A. parasiticus in peanut fields following application to soil. Mycopathologia 151:81-92.

Horn, B. W. 2003. Ecology and population biology of aflatoxigenic fungi in soil. J. Toxicol. Toxin Rev. 22:351-379.

Horn, B. W. 2007. Biodiversity of Aspergillus section Flavi in the United States: A review. Food Addit. Contam. 24:1088-1101.

Horn, B. W., and Dorner, J. W. 1999. Regional differences in production of aflatoxin $\mathrm{B}_{1}$ and cyclopiazonic acid by soil isolates of Aspergillus flavus along a transect within the United States. Appl. Environ. Microbiol. 65: 1444-1449.

Horn, B. W., and Greene, R. L. 1995. Vegetative compatibility within populations of Aspergillus flavus, A. parasiticus, and A. tamarii from a peanut field. Mycologia 87:324-332.

Horn, B. W., Moore, G. G., and Carbone, I. 2009. Sexual reproduction in Aspergillus flavus. Mycologia 101:423-429.

Horn, B. W., Sorensen, R. B., Lamb, M. C., Sobolev, V. S., Olarte, R. A., Worthington, C. J., and Carbone, I. 2014. Sexual reproduction in Aspergillus flavus sclerotia naturally produced in corn. Phytopathology 104:75-85.

Hudson, R. R., and Kaplan, N. L. 1985. Statistical properties of the number of recombination events in the history of a sample of DNA sequences. Genetics 111:147-164.

Huson, D. H., and Bryant, D. 2005. Application of phylogenetic networks in evolutionary studies. Mol. Biol. Evol. 23:254-267.

Jombart, T. 2008. adegenet: A R package for the multivariate analysis of genetic markers. Bioinformatics 24:1403-1405.

Jombart, T. 2015. An introduction to adegenet 2.0.0. http://adegenet.r-forge. r-project.org/files/tutorial-basics.pdf

Jones, R. K., Duncan, H. E., and Hamilton, P. B. 1981. Planting date, harvest date, and irrigation effects on infection and aflatoxin production by Aspergillus flavus in field corn. Phytopathology 71:810-816.

Kamvar, Z. N., Tabima, J. F., and Grünwald, N. J. 2014. Poppr: An R package for genetic analysis of populations with clonal, partially clonal, and/or sexual reproduction. PeerJ 2:e281.

Kepler, R. M., Humber, R. A., Bischoff, J. F., and Rehner, S. A. 2014. Clarification of generic and species boundaries for Metarhizium and related fungi through multigene phylogenetics. Mycologia 106:811-829.

Klich, M. A. 2002. Biogeography of Aspergillus species in soil and litter. Mycologia 94:21-27.

Klich, M. A. 2007. Aspergillus flavus: The major producer of aflatoxin. Mol. Plant Pathol. 8:713-722.

Kuznetsova, A., Brockhoff, P. B., Haubo, R., and Christensen, B. 2016. lmerTest: Tests in linear mixed effects models. R package version 2.0-33.

Lenth, R. V. 2016. Least-squares means: The R package lsmeans. J. Stat. Softw. 69:33.

Linde, C. C., Zala, M., and McDonald, B. A. 2009. Molecular evidence for recent founder populations and human-mediated migration in the barley scald pathogen Rhynchosporium secalis. Mol. Phylogenet. Evol. 51: 454-464.

Liu, Y., and Wu, F. 2010. Global burden of aflatoxin-induced hepatocellular carcinoma: A risk assessment. Environ. Health Perspect. 118:818-824. 
Manabe, M., and Tsuruta, O. 1978. Geographical distribution of aflatoxin producing fungi inhabiting in Southeast Asia. Jpn. Agric. Res. Q. 12: 224-227.

McAlpin, C., Wicklow, D., and Horn, B. 2002. DNA fingerprinting analysis of vegetative compatibility groups in Aspergillus flavus from a peanut field in Georgia. Plant Dis. 86:254-258.

Moore, G. G., Elliott, J. L., Singh, R., Horn, B. W., Dorner, J. W., Stone, E. A., Chulze, S. N., Barros, G. G., Naik, M. K., Wright, G. C., Hell, K., and Carbone, I. 2013. Sexuality generates diversity in the aflatoxin gene cluster: Evidence on a global scale. PLoS Pathog 9:e1003574.

Moore, G. G., Singh, R., Horn, B. W., and Carbone, I. 2009. Recombination and lineage-specific gene loss in the aflatoxin gene cluster of Aspergillus flavus. Mol. Ecol. 18:4870-4887.

Nei, M. 1987. Molecular Evolutionary Genetics. Columbia University Press, New York.

Olarte, R. A., Horn, B. W., Dorner, J. W., Monacell, J. T., Singh, R., Stone, E. A., and Carbone, I. 2012. Effect of sexual recombination on population diversity in aflatoxin production by Aspergillus flavus and evidence for cryptic heterokaryosis. Mol. Ecol. 21:1453-1476.

Peakall, R., and Smouse, P. E. 2006. GENALEX 6: Genetic analysis in Excel. Population genetic software for teaching and research. Mol. Ecol. Resour. 6:288-295.

Probst, C., Bandyopadhyay, R., Price, L. E., and Cotty, P. J. 2011. Identification of atoxigenic Aspergillus flavus isolates to reduce aflatoxin contamination of maize in Kenya. Plant Dis. 95:212-218.

R Core Team. 2017. R: A Language and Environment for Statistical Computing. R Foundation for Statistical Computing, Vienna, Austria.

Rafiei, V., Banihashemi, Z., Jiménez-Díaz, R., Navas-Cortés, J., Landa, B., Jiménez-Gasco, M., Turgeon, B., and Milgroom, M. 2018. Comparison of genotyping by sequencing and microsatellite markers for unravelling population structure in the clonal fungus Verticillium dahliae. Plant Pathol. 67:76-86.

Schuelke, M. 2000. An economic method for the fluorescent labeling of PCR fragments. Nat. Biotechnol. 18:233-234.

Smith, J. M., Smith, N. H., O’Rourke, M., and Spratt, B. G. 1993. How clonal are bacteria? Proc. Natl. Acad. Sci. U.S.A. 90:4384-4388.
Tam, E. W., Chen, J. H., Lau, E. C., Ngan, A. H., Fung, K. S., Lee, K.-C., Lam, C.-W., Yuen, K.-Y., Lau, S. K., and Woo, P. C. 2014. Misidentification of Aspergillus nomius and Aspergillus tamarii as Aspergillus flavus: Characterization by internal transcribed spacer, $\beta$-tubulin, and calmodulin gene sequencing, metabolic fingerprinting, and matrix-assisted laser desorption ionization-time of flight mass spectrometry. J. Clin. Microbiol. 52: 1153-1160.

Tran-Dinh, N., Kennedy, I., Bui, T., and Carter, D. 2009. Survey of Vietnamese peanuts, corn and soil for the presence of Aspergillus flavus and Aspergillus parasiticus. Mycopathologia 168:257-268.

Tran-Dinh, N., Pitt, J. I., and Carter, D. A. 1999. Molecular genotype analysis of natural toxigenic and nontoxigenic isolates of Aspergillus flavus and A. parasiticus. Mycol. Res. 103:1485-1490.

Wickham, H. 2017. Tidyverse: Easily install and load 'Tidyverse' packages. $\mathrm{R}$ package version 1.1.1.

Wicklow, D. T., and Cole, R. J. 1982. Tremorgenic indole metabolites and aflatoxins in sclerotia of Aspergillus flavus: An evolutionary perspective. Can. J. Bot. 60:525-528.

Wicklow, D. T., Dowd, P. F., and Gloer, J. B. 1994. Anti-insectan effects of Aspergillus metabolites. Pages 93-109 in: The Genus Aspergillus. Plenum Press, New York.

Wicklow, D. T., McAlpin, C. E., and Platis, C. E. 1998. Characterization of the Aspergillus flavus population within an Illinois maize field. Mycol. Res. 102:263-268.

Wicklow, D. T., Wilson, D. M., and Nelsen, T. C. 1993. Survival of Aspergillus flavus sclerotia and conidia buried in soil in Illinois or Georgia. Phytopathology 83:1141-1147.

Wild, C. P. 2007. Aflatoxin exposure in developing countries: The critical interface of agriculture and health. Food Nutr. Bull.: S372-S380.

Wild, C. P., and Gong, Y. Y. 2010. Mycotoxins and human disease: A largely ignored global health issue. Carcinogenesis 31:71-82.

Yin, Y., Lou, T., Yan, L., Michailides, T., and Ma, Z. 2009. Molecular characterization of toxigenic and atoxigenic Aspergillus flavus isolates, collected from peanut fields in China. J. Appl. Microbiol. 107: 1857-1865. 